\title{
Entwicklung eines kraftgeregelten Roboterentgratsystems mit Hilfe von Fuzzy-Logik
}

\author{
Mei-Hua Liu. Darmstadt
}

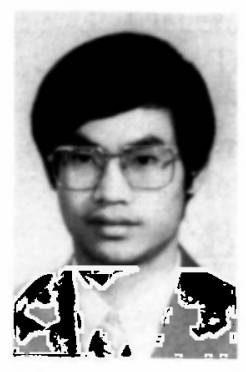

Mei-Hua Liu (Master of Science) ist wissenschaftlicher Mitarbeiter am Institut für Regelungstechnik. Fachgebiet Regelsystemtheorie und Robotik der Technischen Hochschule Darmstadt. Arbeitsgebiete: uissensbasierte. Fuzzy-Logik-gestützte Bearbeitung von Werkstükken mit kraft- und positionsgeregetten Industrierobotern.

Adresse: Technische Hochschule Darmstadt. Institut für Regelungstechnik. Fachgebiel Regelsystemtheorie und Robotik. Landgraf-Georg-Str. 4. D-64283 Darmstadt. Tel. (06151) 165341 .

In diesem Beitrag wird ein auf der Kraftregelung und der Fuzzl-Logik basierendes Roboterentgratkonzept vorgestellt. Ein Algorithmus zur On-line-Erkennung der Positionsungenauigkeiten und der Gratausprägung und. darauf aufsetzend, ein Regelkonzept zur Erzeugung entsprechender Bahnkorrekturen, basierend auf der Normal- und Tangentialkraftregelung, werden entwickelt. Das neu vorgestellte Entgratkonzept und der Kraftregler werden mit Hilfe der Fuzzl-Logik ausgeführt, damit die Reglerparameter an den aktuellen Arbeitspunkt adaptiert werden, die Auswirkung der in Meßwerten enthaltenen Störungen vermindert wird, und das sprunghafte Verhalten beim Ausgleich der Positionsungenauigkeiten verhindert wird. Implementierung und experimentelle Ergebnisse des Entgratkonzeptes werden präsentiert und seine Eigenschaften in Relation zum Einsatz eines konventionellen PID-Reglers diskutiert.

Development of a force-controlled robotic deburring system with the help of fuzzy logic

This paper presents a rohotic deburring strategy based on force feedhack control and fuzzy logic. By controlling the deburring force in the normal and tangential directions of the desired trajectory positional inaccuracies and unknown burr sizes are detected on line and real-time motion corrections are conducted in such a way that the positional inaccuracies are compensated and unknown burr sizes are completely removed. With the use of fuzzy logic control controller parameters are adjusted automatically according to working conditions, disturbances involved in data measurement are suppressed, and smooth behaviour in the compensation of the positional inaccuracies is achieved. Implementation of and experimental results about the proposed deburring strategy are described and its main features are discussed in relation with a conventional PID-Controller.

\section{Einleitung}

Aus funktionellen, ergonomischen und ästhetischen Gründen müssen Werkstücke nach dem Formgebungsoder Bearbeitungsprozeß häufig entgratet werden. In der Industrie wird das Entgraten nach wie vor manuell und häufig unter ungünstigen Umgebungsbedingungen durchgeführt. Seit mehreren Jahren besteht die Bestrebung, den Entgratprozeß durch den Einsatz von sensorunterstützten Industrierobotern zu automatisieren. Bezüglich der Regelung liegen die Schwierigkeiten des Roboterentgratens darin, daß die wechselnde Gratausprägung. der progressive Werkzeugverschleiß und die unvorhersagbaren Positionsungenauigkeiten gleichzeitig erfaßt und die Sensorsignale in Bewegungsänderungen des Roboters in Echtzeit umgesetzt werden müssen. Das erste Problem ist die wechselnde Gratausprägung, deren Größe und Lage nicht vorhersagbar sind. Damit ein Grat ganz entfernt wird, muß die Roboterbewegung so gesteuert oder geregelt werden, da 3 die an verschiedenen Lagen entfernte Werkstolfmenge den jeweiligen Gratausprägungen entspricht. Das zweite Problem sind der Werkzeugverschlei $\beta$ und die Positionsungenauigkeiten, z. B. die Werkstücktoleranzen. der Programmierfehler sowie der Positionierfehler des Werkstückes und des Roboters. Für ein erfolgreiches Entgraten müssen die Durchmesseränderung durch Werkzeugverschleiß und die Positionsungenauigkeiten beim Reglerentwurf berücksichtigt und dadurch beim Entgraten kompensiert werden.

Es zeigt sich. daß die Anpassung der Vorschubgeschwindigkeit an die Gratausprägung durch eine Kraftregelung in der Tangentialrichtung realisiert [1] und [2], und der Ausgleich der Positionsungenauigkeiten und des Werkzeugverschleißes durch eine Kraftregelung in der Normalenrichtung durchgeführt werden kann [3] bis [5]. Weil die Normal- und Tangentialkräfte stark miteinander gekoppelt sind [4] bis [7]. ist es unmöglich, die Normalkraftregelung zum Ausgleich der Positionsungenauigkeiten und des Werkzeugverschleißes und die Tangentialkraftregelung zur Anpassung der Vorschubgeschwindigkeit an die wechselnde Gratausprägung gleichzeitig zu verwenden. Deshalb ist eine Entgratstrategie notwendig. In [8] wurde eine Entgratstrategie entwickelt, nach der sowohl die Geschwindigkeit als auch die Bewegungsbahn gemäß der 
Normalkraft abwechselnd korrigiert werden. Diese Strategie wurde aber in der Praxis nur simuliert. In [9] wurde ein auf der Kraftregelung und der FuzzyLogik basierendes Entgratkonzept vorgestellt, bei dem die Gratausprägung, die Positionsungenauigkeiten und der Werkzeugverschleiß alle gleichzeitig während des Entgratvorgangs durch Kraftregelung erfaßt werden. Eine Entscheidungsstrategie entscheidet aufgrund der Bearbeitungskraft und der Vorschubgeschwindigkeit, wann die Positionsungenauigkeiten und der Werk-

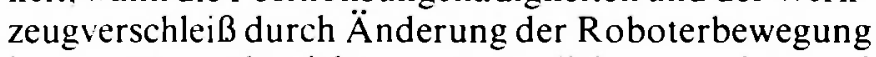
in der Normalenrichtung ausgeglichen werden und wann die Vorschubgeschwindigkeit durch $\ddot{\text { Anderung }}$ der Roboterbewegung in der Tangentialrichtung an die unbekannte Gratausprägung angepaßt werden soll.

In diesem Beitrag werden das in [9] vorgestellte Entgratkonzept weiterentwickelt und seine Eigenschaften in Relation zum Einsatz eines konventionellen PIDReglers diskutiert. Im Abschnitt 2 des Beitrages wird zuerst die Entgratstrategie vorgestellt, wobei die Fuzzy-Logik zur Überwachung der Positionsungenauigkeiten dient. Anschließend wird im Abschnitt 3 der Entwurf des Fuzzy-Reglers dargelegt. Abschnitt 4 präsentiert die Implementierung des Entgratkonzeptes und erörtert seine Eigenschaften. AbschlieBlich erfolgt im Abschnitt 5 eine kurze Zusammenfassung. Überblicke und Grundlagen über Fuzzy-Logik-Regelung findet man in [10] und [11].

\section{Entgratstrategie}

Nach [12] bedeutet Entgraten (deburring), das unerwünschte Werkstoffgebilde, das an Kanten oder auf der Werkstückoberfläche bei der Fertigung entsteht und über die ideale Werkstückoberfläche hinausragt, zu entfernen. Im Bild 1 wird ein typischer Grat an Kanten dargestellt. Sehr oft ist das Entgraten mit dem Abfasen (chamfering) verbunden. Deshalb sollen nicht nur der Grat $\left(\sigma_{g}\right.$ im Bild 1) entfernt, sondern auch eine gewünschte Fase nach dem Entgratvorgang erzeugt werden, d.h., der Spanungsquerschnitt $\sigma$ soll der Summe der Querschnitte der gewünschten Fase $\sigma_{f}$ und des zu entfernenden Grates $\sigma_{g}$ gleichen $\left(\sigma=\sigma_{f}+\sigma_{g}\right)$.

Zwischen der Bearbeitungskraft $f$, dem Spanungsquerschnitt $\sigma$ und der Vorschubgeschwindigkeit $v$ besteht die folgende Beziehung [6], [7] und [9]:

$$
f=c \sigma^{n_{1}} v^{n_{2}} \stackrel{\text {,te }}{=} \phi_{d b}(\sigma, v)
$$

mit $c, n_{1}, n_{2}:$ Konstanten.

Aus (1) kann man erkennen, daß die Bearbeitungskraft durch Änderung des Spanungsquerschnitts oder der Vorschubgeschwindigkeit verändert werden kann. Der Ausgleich der Positionsungenauigkeiten und des Werkzeugverschleißes und die Anpassung der Vorschubgeschwindigkeit an die wechselnde Gratausprägung können deshalb durch eine konstante Kraftregelung verwirklicht werden.

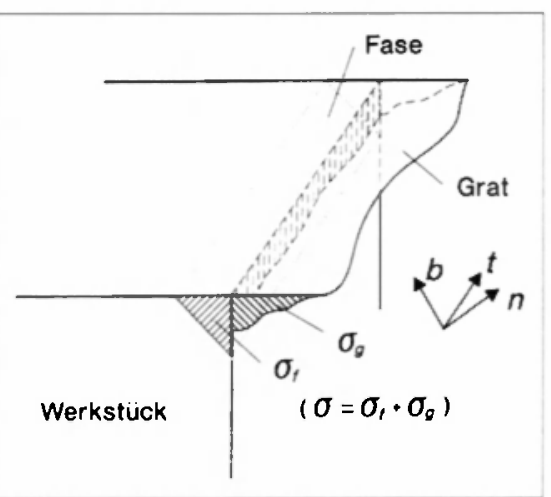

Bild 1: Ein Typischer

Grat an Kanten.

- Zum Ausgleich der Positionsungenauigkeiten und des Werkzeugverschleißes soll die Roboterbewegung in der Normalenrichtung korrigiert werden $(\delta \sigma$-Bewegungskorrektur). Wenn die Vorschubgeschwindigkeit konstant vorgegeben wird und die Bearbeitungskraft konstant durch $\delta \sigma$-Bewegungskorrektur geregelt wird, wird der Spanungsquerschnitt nach (1) auch konstant geregelt. Eine fehlende oder zu kleine Kraft bedeutet, daB das Werkzeug wegen der Positionsungenauigkeiten oder wegen des Werkzeugverschleißes zu weit vom Werkstück entfernt ist; deshalb erzeugt der Kraftregler eine Bewegungskorrektur in der Normalenrichtung so, daß das Werkzeug tiefer ins Werkstück eingreift und der Spanungsquerschnitt und dadurch die Kraft größer wird. Auf der anderen Seite bedeutet eine zu große Kraft, daß das Werkzeug zu tief ins Werkstück schneidet, weshalb der Kraftregler eine Bewegungskorrektur erzeugt, die das Werkzeug etwas aus dem Werkstück hinaus bewegt.

- Zur Anpassung der Vorschubgeschwindigkeit an die wechselnde Gratausprägung soll die Roboterbewegung in der Tangentialrichtung korrigiert werden ( $\delta v$-Bewegungskorrektur), damit die Geschwindigkeit entsprechend der Gratausprägung verändert wird. Aus (1) kann man ersehen, daß ein großer Grat (großer Querschnitt) eine große Bearbeitungskraft erzeugt, wenn die Vorschubgeschwindigkeit konstant bleibt. Je größer der Grat ist, desto größer ist die Kraft. Beim Entgraten mit unbekannter Gratausprägung kann die Anpassung der Geschwindigkeit durch eine auf konstanter Kraftregelung basierende Bewegungskorrektur in der Tangentialrichtung realisiert werden. So wird ein kleiner Grat mit einer hohen Geschwindigkeit und ein großer Grat mit einer niedrigen Geschwindigkeit entfernt, und je größer der Grat wird, desto niedriger wird die Geschwindigkeit.

Um das Problem des Ausgleiches der Positionsungenauigkeiten und des Werkzeugverschleißes und das Problem der Anpassung der Vorschubgeschwindigkeit an die wechselnde Gratausprägung gleichzeitig zu lösen, wird folgende Entgratstrategie eingeschlagen. Es wird angenommen, daß die Größe des kleinsten und des größten möglichen Grates $\left(\sigma_{g \min }\right.$ und $\left.\sigma_{g \max }\right)$ und die Gratgröße am Anfang des Entgratens $\left(\sigma_{g 0}\right)$ bekannt sind. 
Zunächst wird eine Phase zum Ausgleich der Positionsungenauigkeiten und des Werkzeugverschleißes durch konstante Kraftregelung unter $\delta \sigma$-Bewegungskorrektur vor dem Entgratvorgang eingeführt. In dieser Phase wird die Vorschubgeschwindigkeit konstant gehalten $\left(l=v_{0}\right)$ und die Bearbeitungskraft nach der Sollkraft $f_{d}$ geregelt. Die Größe der Sollkraft bestimmt. wie tief das Werkzeug ins Werkstück einschneidet. d.h. der gewünschte Spanungsquerschnitt am Anfang $\left(\sigma_{0}\right)$ kann durch die Kraftregelung unter $\delta \sigma$-Bewegungskorrektur eingestellt werden. obwohl die Positionsungenauigkeiten und der Werkzeugverschleiß vorhanden sind. Die Sollkraft soll entsprechend der gewünschten Fase $\sigma_{f}$ und der Gratgröße am Anfang $\sigma_{30}$ wie folgt vorgegeben werden:

$$
f_{d}=\phi_{d b}\left(\sigma_{0}, v_{0}\right)
$$

mit $\sigma_{0}=\sigma_{f}+\sigma_{g 0}$

Man kann aus (2) erkennen: Mit einer vorgegebenen Vorschubgeschwindigkeit $v_{0}$ und einer bestimmten Gratgröße $\sigma_{g 0}$ ist der Querschnitt der erzeugten Fase desto größer, je größer die Sollkraft ist.

Nachdem die Bearbeitungskraft $f$ in einen gewünschten Bereich eingeregelt worden ist:

$$
\begin{aligned}
& |e(k-m)|, \mid e(k-m+1 ; \mid, \ldots, \\
& |e(k-1)|,|e(k)|<\eta_{1},
\end{aligned}
$$

wobei $e(k)=f_{d}-f(k)$ die Kraftabweichung dargestellt und $k$ die aktuelle Zeit und $m$ eine ganze Zahl sind, sind die Positionsungenauigkeiten und der Werkzeugverschleiß ausgeglichen. Zu diesem Zeitpunkt wird von der $\delta \sigma$-Bewegungskorrektur auf die $\delta v$-Bewegungskorrektur umgeschaltet und der wirkliche Entgratvorgang beginnt. Infolge der konstanten Kraftregelung unter $\delta v$-Bewegungskorrektur wird die Vorschubgeschwindigkeit nun erhöht, wenn ein kleiner Grat vorliegt, und reduziert, wenn ein großer Grat auftritt. Auf diese Weise wird ein unbekannter Grat mit einer entsprechenden Vorschubgeschwindigkeit entfernt.

In vielen Fällen sind die Positionsungenauigkeiten und der Werkzeugverschleiß variabel, und deshalb müssen sie während des Entgratvorgangs überwacht und eventuell erneut kompensiert werden. $\mathrm{Zu}$ diesem Zweck werden eine Minimal- und eine Maximalvorschubgeschwindigkeit ( $V_{\min }$ und $V_{\max }$ ) eingeführt. Die Geschwindigkeiten $V_{\min }$ und $V_{\max }$ entsprechen den Vorschubgeschwindigkeiten, mit denen der größte und der kleinste zulässige Spanungsquerschnitt mit der Sollkraft erzeugt werden. Der größte Spanungsquerschnitt $\sigma_{\max }$ ist der Querschnitt der gewünschten Fase plus dem Querschnitt des größten möglichen Grates $\left(\sigma_{\max }=\sigma_{f}+\sigma_{g \max }\right)$, und deshalb ist die kleinste Vorschubgeschwindigkeit $V_{\min }$ aus $f_{d}=\phi_{d b}\left(\sigma_{\max }, V_{\min }\right) \mathrm{zu}$ ermitteln. Ganz ähnlich wird die größte Vorschubgeschwindigkeit $V_{\max }$ aus $f_{d}=\phi_{d b}\left(\sigma_{\min }, V_{\max }\right)$ berechnet. wobei $\sigma_{\min }$ den kleinsten Spanungsquerschnitt darstellt $\left(\sigma_{\text {min }}=\sigma_{f}+\sigma_{g \text { min }}\right)$. Außerdem wird ein zulässiger Bereich $\left(|e|<\eta_{2}\right)$ für die Bearbeitungskraft definiert.
Wenn eine der folgenden Bedingungen erfüllt wird: $v \leq V_{\min }, v>V_{\max }$ und $|e|>\eta_{2}$, dann werden die Positionsungenauigkeiten oder der Werkzeugverschleif neu festgestellt, und sie müssen durch $\delta \sigma$-Bewegungskorrektur erneut kompensiert werden.

Der Erfülltheitsgrad der $v$-verbundenen Bedingungen $\mu(v)$ und jener der $|e|$-verbundenen Bedingung $\mu(|e|)$ werden wie folgt definiert:

$$
\begin{aligned}
& \mu(v)= \begin{cases}1 & \text { wenn } v<V_{\min }, \operatorname{oder} v>V_{\max } \\
0 & \text { sonst. }\end{cases} \\
& \mu(|e|)= \begin{cases}1 & \text { wenn }|e|>\eta_{2} \\
0 & \text { sonst. }\end{cases}
\end{aligned}
$$

Die Notwendigkeit für den Ausgleich kann durch einen Notwendigkeitsgrad $\mu_{n}$ definiert werden, der den Wert 1 annimmt, wenn eine der oben dargestellten Bedingungen erfüllt wird. und den Wert 0 annimmt. wenn keine der Bedingungen erfüllt wird. Dann erhält man: $\mu_{n}=\max \{\mu(v), \mu(|e|)\}$. Wenn $\mu_{n}=1$ ist. so haben sich die Positionsungenauigkeiten oder der Werkzeugverschleiß verändert und deshalb soll die $\delta \sigma$-Bewegungskorrektur erneut verwendet werden. Wenn $\mu_{n}=0$ ist. so haben sich die Positionsungenauigkeiten und der Werkzeugverschleiß nicht verändert und deshalb geht der Entgratvorgang ohne die $\delta \sigma$-Bewegungskorrektur weiter.

Die drei Bedingungen und die mit ihnen verbundenen Erfülltheitsgrade sind im Bild 2(a) dargestellt. Aus diesem Bild ist leicht zu erkennen. daß die oben dargestellte Entgratstrategie sehr störempfindlich ist. $\mathrm{Ob}$ die Positionsungenauigkeiten oder der Werkzeugverschleiß erneut kompensiert werden, hängt davon ab. ob ein bestimmter Wert der Vorschubgeschwindigkeil oder der Kraftabweichung erreicht wird. Deshalb können sprunghafte Schlußfolgerungen infolge einer geringen Anderung der Meßwerte auftreten. Um das zu verhindern, definiert man einen Übergang zwischen den extremen Zuständen. wie es im Bild 2(b) dargestellt ist Dieser Übergang kann mit Hilfe der Fuzzy-Logik sehı einfach formuliert werden. Dazu definiert man zwei linguistische Terme für die Vorschubgeschwindigkeit: $Z K$

Bild 2: Bedingungen für die Erkennung der Positionsungenauigkei ten und des Werkzeugverschleißes.
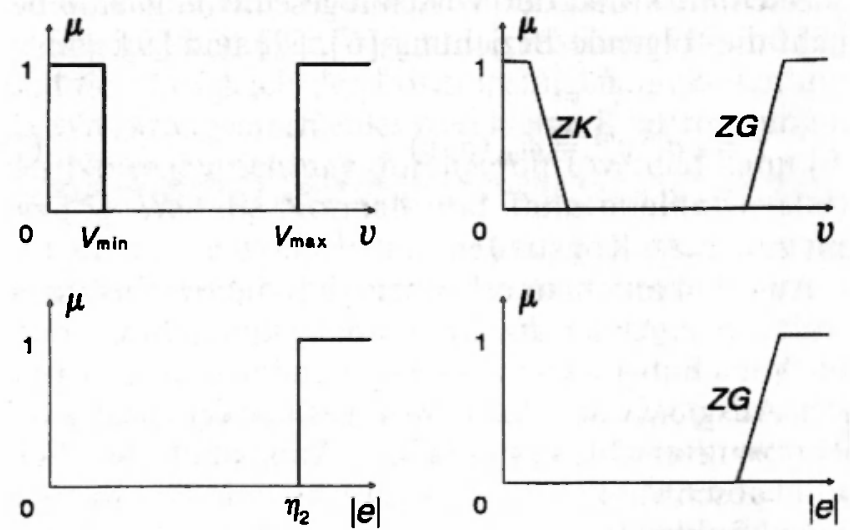

(a)

(b) 
(zu klein) und $Z G$ (zu groß) und einen Term für die Kraftabweichung: $Z G$ (zu groß). Die Notwendigkeit für einen erneuten Ausgleich der Positionsungenauigkeiten und des Werkzeugverschleißes wird dann durch die folgende Regel dargestellt:

WENN $v=Z K$ ODER $v=Z G$ ODER $|e|=Z G$.

DANN ist der Ausgleich erneut notwendig.

Der Notwendigkeitsgrad für den Ausgleich ist dann gleich dem Erfülltheitsgrad der Antezedenzien in Regel (6). der wie folgt berechnet werden kann:

$$
\mu_{n}=\max \left\{\mu_{Z K}(v), \mu_{Z G}(v), \mu_{Z G}(|e|)\right\},
$$

wobei $\mu_{Z K}(v), \mu_{Z G}(v)$, und $\mu_{Z G}(|e|)$ in der Reihenfolge den Zugehörigkeitsgrad von $v$ zu $Z K$, von $v$ zu $Z G$ und von $|e|$ zu $Z G$ kennzeichnet. Im Bild 2(b) kann man sehen. da $\beta \mu_{Z K}(v), \mu_{Z G}(v)$. und $\mu_{Z G}(|e|)$ alle Werte von 0 bis 1 haben können. Deshalb kann $\mu_{n}$ auch alle möglichen Werte zwischen 0 und 1 annehmen und deswegen wird ein Übergang zwischen keinem Ausgleich und vollem Ausgleich gebildet. Dieser verhindert, daß eine durch eine Störung verursachte kleine Änderung der Vorschubgeschwindigkeit oder der Bearbeitungskraft zu einem Sprung von keinem Ausgleich zu vollem Ausgleich führt.

Einen wichtigen Sonderfall der Entgratstrategie kann man ableiten, wenn kein Grat am Anfang des Entgratvorgangs vorhanden ist. In diesem Fall wird die Schnittiefe in der anfänglichen Phase ganz nach der gewünschten Fase eingestellt, und während des wirklichen Entgratvorgangs danach wird die Vorschubgeschwindigkeit proportional zur Gratgröße erniedrigt. Je größer ein Grat ist, desto mehr wird die Vorschubgeschwindigkeit reduziert. An den Stellen, wo keine Grate vorhanden sind, nimmt der Spanungsquerschnitt seinen kleinsten Wert, den Querschnitt der gewünschten Fase, an: $\sigma_{\min }=\sigma_{f}$. Deshalb entspricht die Maximalvorschubgeschwindigkeit der Geschwindigkeit, mit der die gewünschte Fase mit der Sollkraft $f_{d}$ erzeugt wird, falls keine Grate vorhanden sind: $V_{\max }=v_{0}$. in praktischen Situationen sollte $V_{\max }$ etwas größer als $v_{0}$ angesetzt werden, um Störungen zu unterdrücken.

\section{Fuzzy-Regler}

Im Abschnitt 2 wurde eine Entgratstrategie dargestellt, nach der eine anfängliche Phase vor dem Entgratvorgang zum Ausgleich der Positionsungenauigkeiten und des Werkzeugverschleißes unter $\operatorname{der} \delta \sigma$-Bewegungskorrektur eingeführt wird und während des Entgratvorgangs unter der $\delta v$-Bewegungskorrektur die Positionsungenauigkeiten und der Werkzeugverschlei $\beta$ überwacht und gegebenenfalls erneut durch die $\delta \sigma$-Bewegungskorrektur kompensiert werden. In diesem Abschnitt wird ein Fuzzy-Regler vorgestellt, mit dem die benötigten Bewegungskorrekturen erzeugt werden. Die wichtigsten Anregungen für die Verwendung der Fuzzy-Logik sind:
1. das dynamische Verhalten des Entgratprozesses ist nichtlinear und die Reglerparameter sollen gemä $B$ dem aktuellen Arbeitspunkt eingestellt werden;

2. das dynamische Modell des Entgratenprozesses ist sehr komplex und genaue mathematische Modelle sind nicht erhältlich;

3. die Meßwerte aus dem $\mathrm{Kraft} /$ Momenten-Sensor sind wegen der Störungen schwankend und die in den Meßwerten enthaltenen Störungen sollen beim Reglerentwurf berücksichtigt werden.

Zur automatischen Anpassung der Reglerparameter an den aktuellen Arbeitspunkt werden die Reglerverstärkungen mit Hilfe von Fuzzy-Regeln nach der Vorschubgeschwindigkeit eingestellt, weil die Vorschubgeschwindigkeit einen der wichtigsten der Parameter darstellt, die die Arbeitsbedingungen widerspiegeln. Zum Beispiel kann eine kleine Änderung des Spanungsquerschnitts eine große Änderung der Bearbeitungskraft verursachen wenn die Vorschubgeschwindigkeit sehr groß ist. Wenn sie jedoch sehr klein ist, wird eine große Änderung des Spanungsquerschnitts benötigt, um dieselbe Kraftänderung zu produzieren. Die konventionelle Methode zur Unterdrückung der in Meßwerten enthaltenen Störung ist die Verwendung eines Digitalfilters, die aber gleichzeitig eine große Zeitverzögerung ins System einführt. Die Zeitverzögerung ist ein ernsthaftes Problem für schnelle Prozesse wie Entgraten. Oft muß man einen Kompromiß zwischen Genauigkeit und Phasenverschiebung treffen. Mit Hilfe der Fuzzy-Logik wird die Störung direkt beim Reglerentwurf berücksichtigt und dadurch wird eine Zeitverzögerung verhindert.

Das Grundziel des Reglerentwurfs ist es, die Kraftabweichung auszuregeln. Deshalb werden die gewünschten Bewegungskorrekturen $\delta \sigma_{d}$ und $\delta v_{d}$ als Funktionen der Kraftabweichung entworfen:

$$
\begin{aligned}
& \delta \sigma_{d}(k)=\alpha_{1}(k) \mathscr{G}[\tilde{e}(k)], \\
& \delta v_{d}(k)=\alpha_{2}(k) \mathscr{G}[\ddot{e}(k)],
\end{aligned}
$$

mit $\mathscr{G}[\hat{e}(k)]$ : zu entwerfende Fuzzy-Beziehung,

$$
\mathscr{G}[\dot{e}(k)] \in[-\mathscr{D}, \mathscr{D}] ;
$$

$\dot{e}(k)$ : normalisierte Kraftabweichung,

$$
\begin{aligned}
& \dot{e}(k)=\mathscr{K}_{e} e(k) \in[-\mathscr{D}, \mathscr{D}], \\
& \mathscr{K}_{e}: \text { Skalierungsfaktor; }
\end{aligned}
$$

$\alpha_{1}(k), \alpha_{2}(k)$ : Regler-Verstärkungen.

Auf dem normalisierten Universum $[-\mathscr{D}, \overline{\mathscr{D}}]$ werden sieben Fuzzy-Terme mit dreieckigen Zugehörigkeitsfunktionen definiert: $N G$ (negativ groß), $N M$ (negativ mittelmäßig), $N K$ (negativ klein), $N L$ (null), $P K$ (positiv klein), $P M$ (positiv mittelmäßig), und $P G$ (positiv groß), wie im Bild 3(a) dargestellt. Der Fuzzy-Regelungsalgorithmus wird durch eine Reihe von FuzzyProduktionsregeln definiert:

$$
\begin{aligned}
\mathscr{R}_{i}: & \operatorname{WENN} \bar{e}(k)=\mathscr{A}_{i}, \operatorname{DANN} \mathscr{G}[e(k)]=\mathscr{B}_{i} ; \\
& i=1, \ldots, 7,
\end{aligned}
$$

wobei $\mathscr{A}_{i}$ und $\mathscr{B}_{i}$ einen jeweils für $e(k)$ und $\mathscr{G}[\tilde{e}(k)]$ 
(a)

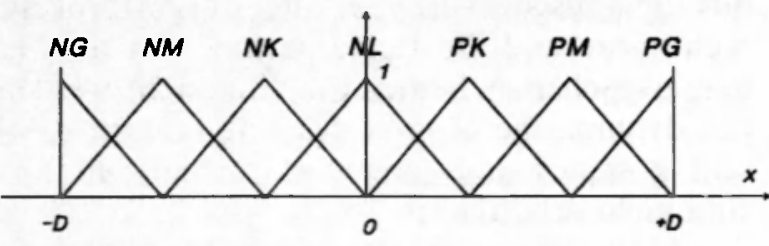

(b)

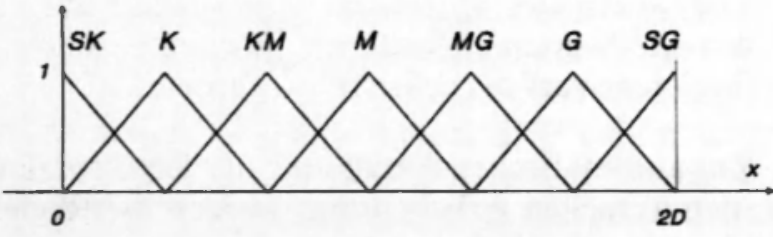

Bild 3: Zugehörigkeitsfunktionen

definierten Fuzzy-Term bezeichnen. Die einzelnen Fuz$z y$-Regeln werden in Tabelle 1 zusammengefaßt. Man kann aus der Tabelle erkennen, daß eine proportionale Verbindung zwischen dem Ein- und dem Ausgang des Reglers gebildet wird, die das Verhalten eines konventionellen P-Reglers aufweist.

Tabelle 1: Fuzzy-Regeln für Kraftregelung.

\begin{tabular}{|c|ccccccc|}
\hline $\bar{e}(k)$ & $N G$ & $N M$ & $N K$ & $N L$ & $P K$ & $P M$ & $P G$ \\
\hline $\mathcal{G}[\tilde{e}(k)]$ & $N G$ & $N M$ & $N K$ & $N L$ & $P K$ & $P M$ & $P G$ \\
\hline \hline$v(k)$ & $S K$ & $K$ & $K M$ & $M$ & $M G$ & $G$ & $S G$ \\
\hline $\mathcal{F}_{1}[\tilde{v}(k)]$ & $S G$ & $G$ & $M G$ & $M$ & $K M$ & $K$ & $S K$ \\
\hline \hline$\tilde{v}(k)$ & $S K$ & $K$ & $K M$ & $M$ & $M G$ & $G$ & $S G$ \\
\hline $\mathcal{F}_{2}[\tilde{v}(k)]$ & $S K$ & $K$ & $K M$ & $M$ & $M G$ & $G$ & $S G$ \\
\hline
\end{tabular}

Jede Regel von (10) liefert eine Stellgröße, die in Form eines Fuzzy-Teams dargestellt wird. Der Erfülltheitsgrad $\mu_{t}$ von Regel $\mathscr{R}_{1}$ ist durch den Wahrheitswert von $\tilde{e}(k)=\mathscr{x}_{i}$, oder durch den Zugehörigkeitsgrad von $\dot{e}(k) \mathrm{zu} \varsigma_{x_{i}}$, gegeben, nämlich $\mu_{i}=\mu_{\text {. }}[\dot{e}(k)]$. Bei der Berechnung der Wahrheitswerte wird die Schwankung der Meßwerte durch eine Meßwert-Toleranz $\pm \varepsilon$ berücksichtigt (Bild 4). Die defuzzifizierte gesamte Stellgröße wird nach der Höhenmethode [13] berechnet:

$$
\mathscr{G}[\dot{e}(k)]=\sum_{i=1}^{7} \mu_{i} \gamma_{i}^{c} / \sum_{i=1}^{7} \mu_{i},
$$

wobei $\gamma_{i}$ der defuzzifizierte (oder zentrale) Wert von $\mathscr{B}_{i}$ ist.

Wie vorher erwähnt, ist das dynamische Verhalten des Entgratprozesses nichtlinear und von den Arbeits-

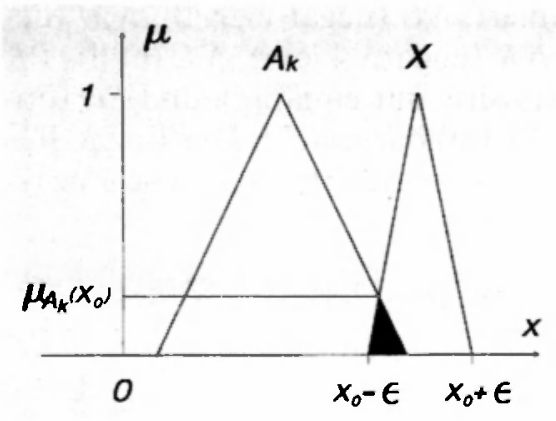

Bild 4:

Berücksichtigung der Meßwertschwankung bedingungen abhängig. Deshalb sollen die Reglerparameter gemäß dem aktuellen Arbeitspunkt eingestellt werden. Unter Berücksichtigung der Tatsache. daß die Vorschubgeschwindigkeit einen der wichtigsten der $\mathrm{Pa}$ rameter darstellt, die die Arbeitsbedingungen widerspiegeln, werden die Reglerverstärkungen wie folgt an die Vorschubgeschwindigkeit adaptiert:

$$
\begin{aligned}
& x_{1}(k)=a_{1} \cdot \mathscr{F}_{1}[\hat{v}(k)]+h_{1} . \\
& x_{2}(k)=a_{2} \cdot \mathscr{F}_{2}[\bar{v}(k)]+h_{2},
\end{aligned}
$$

$\operatorname{mit} \tilde{F}_{1}[\tilde{v}(k)], \mathscr{F}_{2}[\tilde{v}(k)]$ :

zu entwerfende Fuzzy-Beziehungen. $\mathscr{F}_{1}[\dot{i}(k)] . \dot{F},[\bar{i}(k)] \in[0.2 \mathscr{Y}]$;

$i(k)$ :

normalisierte Vorschubgeschwindigkeit.

$\tilde{v}(k)=\mathscr{K}_{\mathrm{r}} v(k)+\mathscr{K}_{c \cdot 0} \in[0,2 \mathscr{L}]$

$a_{1}, a_{2}, h_{1}, h_{2}, \mathscr{K}_{i}, \mathscr{K}_{\mathrm{v} 0}:$

Skalierungsfaktoren.

Auf dem normalisierten Universum $[0,2 \mathscr{L}]$ werden sieben Fuzzy-Terme mit dreieckigen Zugehörigkeitsfunktionen definiert: $S K$ (sehr klein), $K$ (klein), $K M$ (klein-mittelmäßig), $M$ (mittelmäßig), $M G$ (mittelmäBig-groß), $G$ (groß), und $S G$ (sehr groß), wie im Bild 3(b) dargestellt. Die Fuzzy-Beziehungen $\tilde{F}_{1}[\tilde{v}(k)]$ und $\tilde{\mathcal{F}}_{2}[\tilde{v}(k)]$ werden wic in $(10)$ von einer Reihe von Fuzzy-Produktionsregeln generiert, und die Produktionsregeln werden ebenfalls in Tabelle 1 gegeben, z. B., man kann aus der Tabelle folgende Regel ablesen: ,WENN $\dot{v}(k)=S K$. DANN $\mathscr{F}_{1}[\tilde{v}(k)]=S G^{\prime \prime}$. Die defuzzifizierten Größen aus den Fuzzy-Beziehungen $\tilde{F}_{1}[\tilde{v}(k)]$ und $\tilde{\mathscr{F}}_{2}[\bar{v}(k)]$ werden ähnlich wie (11) nach der Höhenmethode der Defuzzifizierung berechnet. Die Reglerverstärkungen werden nach (12) und (13) bestimmt. Die Bewegungskorrekturen werden durch (8) und (9) gegeben und nach der im Abschnitt 2 vorgestellten Entgratstrategie durchgeführt.

\section{Implementierung und Diskussion}

Das als Experiment aufgebaute Entgratsystem besteht aus einem Industrieroboter Manutec $r 3$, einer Robotersteuerung RCM 3, einem Regler-Rechner, einem DFVLR 6-DOF Kraft/Momenten-Sensor, und einem druckluftgetriebenen Entgratwerkzeug, wie im Bild 5 dargestellt. Eine ausführliche Beschreibung der einzelnen Komponenten und des praktischen Verfahrens zur Realisierung der Kraftregelung auf dem positionsgeregelten Industrieroboter findet man in [9].

Experimente wurden mit Werkstücken aus Kunststoff und Aluminium durchgeführt. Die Bilder 6 und 7 zeigen eine Bearbeitungskraft und eine Vorschubgeschwindigkeit, die während des Entgratens einer linearen Kunststoff-Kante mit einem trapezoiden Grat aufgenommen wurden. Das Werkstück wurde absichtlich mit einem Fehler in der Normalenrichtung der linearen Kante positioniert, um den Ausgleich von Positionsungenauigkeiten zu prüfen. Durch $\delta \sigma$-Korrektur 


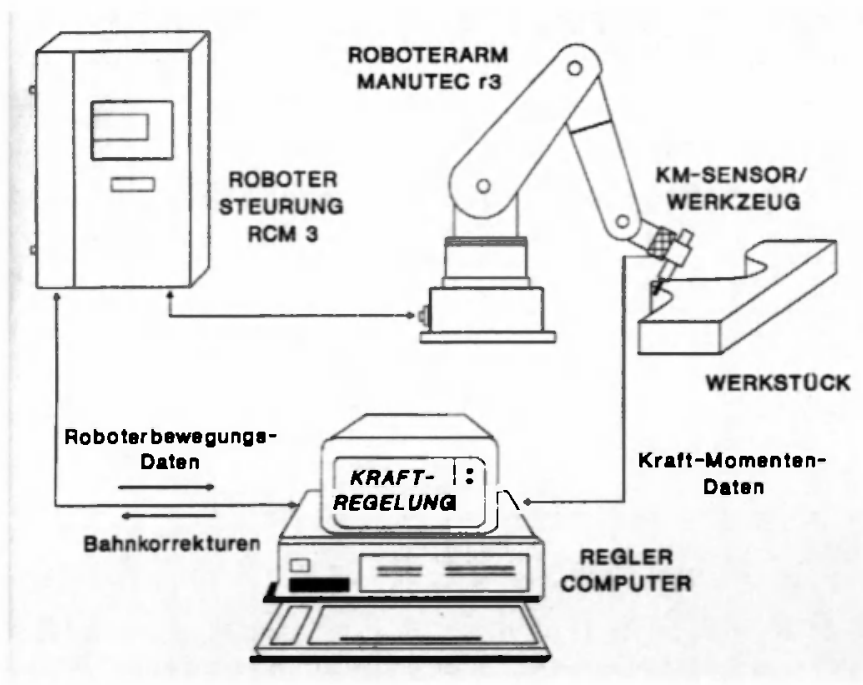

Bild 5: Aufbau des Entgratsystems.

wurde der Positionierfehler zuerst kompensiert und danach der trapezoide Grat durch entsprechende Vorschubgeschwindigkeit entfernt. Die automatische Anpassung der Vorschubgeschwindigkeit an die Gratveränderung ist im Bild 7 deutlich zu sehen.

Die Verwendung der Fuzzy-Logik in diesem Beitrag geht auf die praktische Situation und die spezifische Problemdarstellung zurück. Die Fuzzy-Logik erweist sich als ein geeignetes Mittel zur Darstellung des Übergangs beim Ausgleich der Positionsungenauigkeiten und des Werkzeugverschleißes, zur Berücksichtigung der Meßwertschwankung und zur Einstellung der Reglerparameter gemäß dem Arbeitspunkt. Theoretisch kann man zwar die Entgratstrategie und den Regelalgorithmus in einer anderen Weise darstellen, aber die Darstellung mit Hilfe der Fuzzy-Logik ist viel einfacher und kompakter. Im Vergleich mit dem konventionellen PID-Regler hat der Fuzzy-Ansatz folgende Vorteile:

1. Beim Fuzzy-Ansatz werden die etwas schwankenden Meßwerte direkt zum Regler geführt und die Meßwertschwankung wird im Regler selbst berücksichtigt. Dadurch entsteht keine zusätzliche Zeitverzögerung, die sonst durch Digitalfilter unvermeidbar ist.

Bild 6: Bearbeitungskraft.

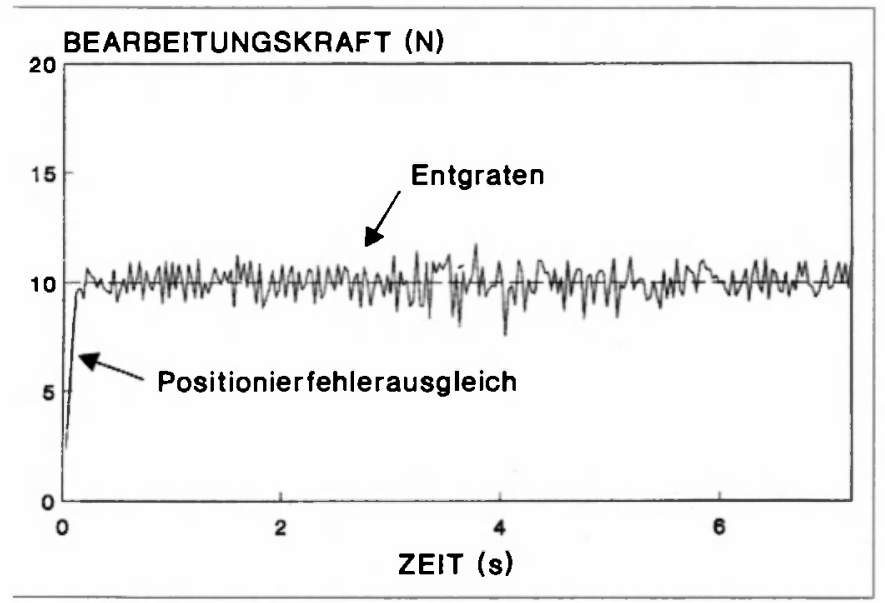

2. Die Reglerverstärkungen werden automatisch gemäß der Vorschubgeschwindigkeit eingestellt. Dadurch wird das nichtlineare Verhalten des Entgratprozesses in einer einfachen Weise berücksichtigt und die Reglerparameter werden an den aktuellen Arbeitspunkt angepaßt.

3. Mit der Fuzzy-Logik wird der Ausgleich der Positionsungenauigkeiten und des Werkzeugverschleißes in einer glatten Weise durchgeführt. Ein Übergang zwischen keinem Ausgleich und vollem Ausgleich wird eingeführt. Dadurch wird verhindert. daß eine durch Störungen verursachte kleine Änderung der Vorschubgeschwindigkeit oder der Bearbeitungskraft zu einem Sprung führt.

Die Bilder 8 und 9 stellen ein Beispiel aus zwei Entgratversuchen unter absichtlich vorgegebenem Programmierfehler dar: der eine mit dem hier dargestellten weichen Übergang, der andere ohne diesen Übergang. Man kann deutlich sehen, daß der Ansatz mit der Fuzzy-Logik besser funktionierte.

Die mit der Fuzzy-Logik verbundenen Nachteile sind folgende:

1. Der Entwurfsvorgang ist beim Fuzzy-Ansatz zeitaufwendiger und komplizierter als beim PID-Regler. Die Anzahl der zu definierenden linguistischen Terme und ihre Zugehörigkeitsfunktionen, die Skalierungsfaktoren, und vor allem die Produktionsregeln müssen oft durch Versuch und Irrtum festgelegt werden. Hilfsmittel dabei sind die erfolgreichen Anwendungen in der Literatur [10] und theoretische Analysen und praktische Untersuchungen des Entgratprozesses [9] und [15].

2. Im Hinblick auf den Aufwand der Implementierung ist der Ansatz mit der Fuzzy-Logik auch viel aufwendiger und komplizierter als ein PID-Regler. Das bedeutet auch, daß die verbesserten Eigenschaften durch eine kompliziertere Implementierung erzielt werden.

Der Ansatz mit der Fuzzy-Logik läßt sich auch als zeitvarianter nichtlinearer P(ID)-Regler auffassen. Der Regler wird auf einem einfachen P-Regler aufgebaut

Bild 7: Vorschubgeschwindigkeit.

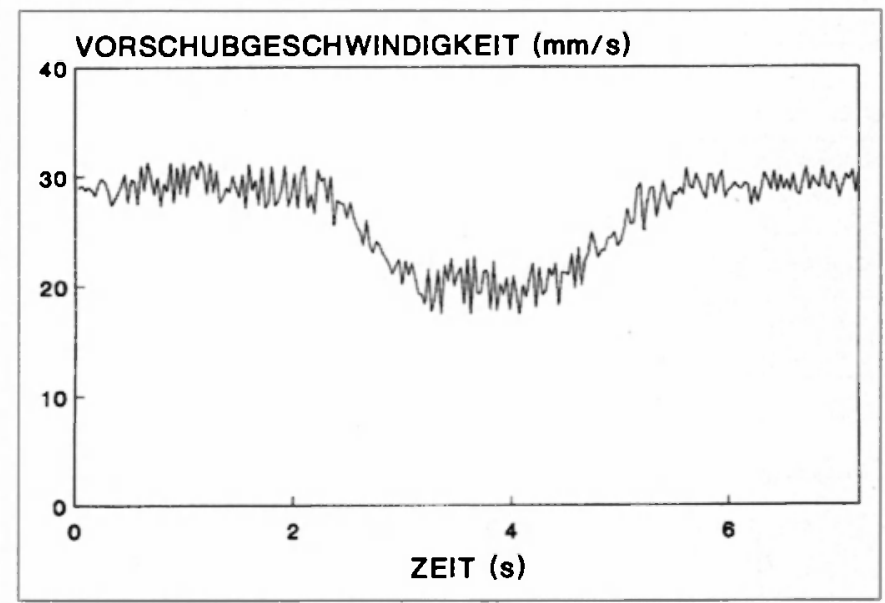




\section{Z-KOORDINATE DES WERKZEUGS (mm)}

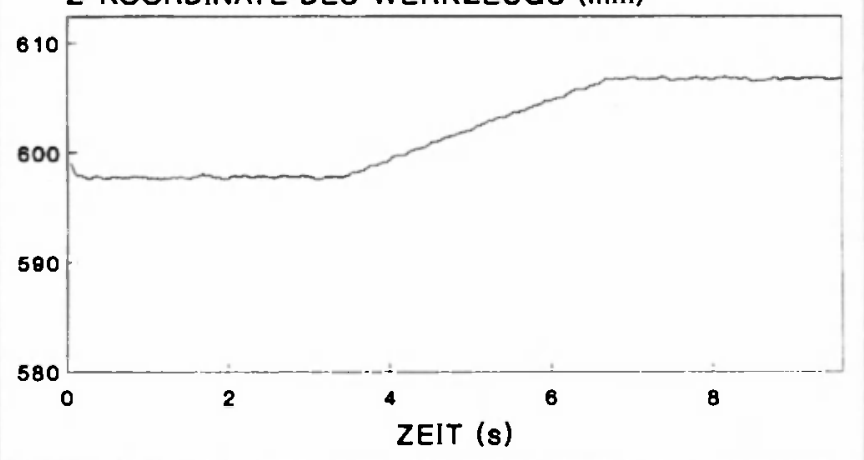

Bild 8: Entgraten mit weichem Übergang.

und durch Änderung der Reglerverstärkung und Einbau nichtlinearer Elemente zwischen dem Ein- und Ausgang erweitert. Mit Hilfe dieser Anschauung kann man dann gut verstehen, warum der Ansatz mit FuzzyLogik für das spezifische Problem besser funktioniert. aber komplizierter ist, als ein reiner P-Regler.

\section{Zusammenfassung}

In diesem Beitrag wurde ein auf der Fuzzy-Logik und Kraftregelung basierendes Entgratkonzept vorgestellt. Ein Algorithmus zur On-line-Erkennung der Positionsungenauigkeiten und der Gratausprägung und. darauf aufsetzend, ein Regelkonzept zur Erzeugung entsprechender Bahnkorrekturen wurden entwickelt. Dabei wurde die Fuzzy-Logik sowohl bei der EchtzeitRegelung der Bearbeitungskraft als auch bei der Echtzeit-Überwachung der Positionsungenauigkeiten erfolgreich eingesetzt. Das neu vorgestellte Entgratkonzept unter Einsatz der Fuzzy-Logik wurde vom Prinzip her mit dem konventionellen PID-Regler verglichen: es zeigten sich folgende Vorteile:

1. Der Fuzzy-Regler behandelt die gestörten Meßwerte, ohne daß ein zusätzlicher Digitalfilter eingebaut werden muß.

2. Ein Übergang wird beim Ausgleich der Positionsungenauigkeiten und des Werkzeugverschleißes durch Wahl geeigneter Zugehörigkeitsfunktionen gebildet und deshalb werden sprunghafte Aktionen infolge einer geringen Änderung der Meßwerte verhindert.

3. Das nichtlineare Verhalten des Entgratprozesses ist durch eine Anpassung der Reglerparameter an den Arbeitspunkt berücksichtigt.

Nachteile des Ansatzes mit der Fuzzy-Logik sind der zeitaufwendigere Entwurfsvorgang und die kompliziertere Software-Implementierung. Einige experimentelle Ergebnisse wurden vorgestellt. Weitere Entwicklung des Roboterentgratsystems und weitere Anwendungen der Fuzzy-Logik findet man in [14] und [15].

\section{Danksagung}

Der Autor dankt der Firma ISRA Systemtechnik für die Unterstützung bei der experimentellen Untersuchung des vorgestellten Entgratkonzeptes, insbesondere Herrn Dipl.-Ing. E. Ersü (dem Ge-

\section{Z-KOORDINATE DES WERKZEUGS (mm)}

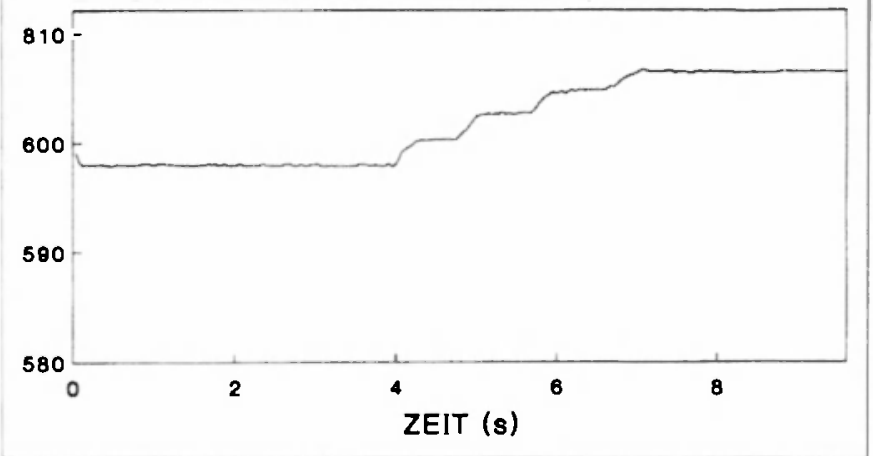

Bild 9: Entgraten ohne weichen Übergang.

schäftsführer). Herrn Dipl.-Ing. S. Wienand (dem Bereichsleiter Robotik) und Herrn Dipl.-Ing. A. Seyffer (dem Bereichsleiter LRCS).

\section{Literatur}

[1] Plank, G., und Hirzinger, G.: Controlling a robots motion speed by a force-torque-sensor for deburring problems. Proc. of the 4th IFAC IFIP Symp. on Infor. Contr. Problems in Manufacturing Technology (1982), S. 97-102.

[2] Kazerooni, H.: Automated robotic deburring using impedance control. IEEE Control System Magazine (1987). S. 21-25.

[3] Stepien, T. M., Sweet, L. M., Good, M.C., und Tomizuka, M. Control of tool/workpiece contact force with application to robotic deburring. IEEE $J$. of Robotics and Automation RA-3 (1987). S. 7-18

[4] Corke, P.I. Roberts, M. V. Kirkham, R.J., und Good, M. C.: Force-controlled robotic deburring. Proc. of the Int. Symp. and Exposition on Robots. Robots: Coming of Age (1988). S. $817-828$

[5] Bone, G.M., Elhestawi, M.A., Lingarkar, R., und Liu, L. Force control for robotic deburring. Trans. ASME: J. DSMC 113 (1991). S. 395-400.

[6] Bone, G. M., und Elbestawi, M.A.: Robotic force control for deburring using an active end effector. Robotica 7 (1989). S. $303-308$

[7] Schulz, M.: Beitrag zum Einsatz von Industrierobotern beim Entgraten von Aluminiumguß. Düsseldorf, V.DI-Verlag 1988.

[8] Puls, F. M., und Barash. M. M.: An adaptive control algorithm for robotic deburring. Journal of Manufacturing Systems (1985), S. 169-178

[9] Liu, $M_{-}-H_{.}:$Robotic deburring based on fuzzy force control. Proc. IEEE/RSJ Int. Conf. on Intelligent Robots and Systems (1992), S. 782-789

[10] Lee, C.C.: Fuzzy logic in control systems: fuzzy logic controller. IEEE Trans. on Systems. Man and Cybern. SMC-20 (1990). S. 404-435.

[11] Zimmermann. H.-J.: Fuzzy set theory - and its applications. 2. Aufl., Boston. Dordrecht. London Kluwer Academic Publishers 1991.

[12] Schäfer, F: Entgraten: Theorie. Verfahren. Anlagen. Mainz, Verlag: Krausskopf 1975.

[13] Mizumoto, $M_{\text {.: }}$ Improvement methods of fuzzy controls. Proc. of the 3rd IFSA (Int. Fuzzy Systems Association) World Congress (1989). S. 60-62.

[14] Liu. M.-H., und Wienand. S.: Applications of the fuzzy logic in automated robotic deburring. In: International Journal Fuzzy Sets and Systems 1994

[15] Liı, M.-H: Fuzzy-Modellbildung für automatisiertes Roboterentgraten. Proc. 3. Workshop ..Fuzzy Control" des GMA-UA 1.4.2. Dortmund 1993. S. 141-156.

Manuskripteingang: 5. Februar 1993.

Verfügbar unter

lediglich die vom Gesetz vorgesehenen Nutzungsrechte gemäß UrhG 


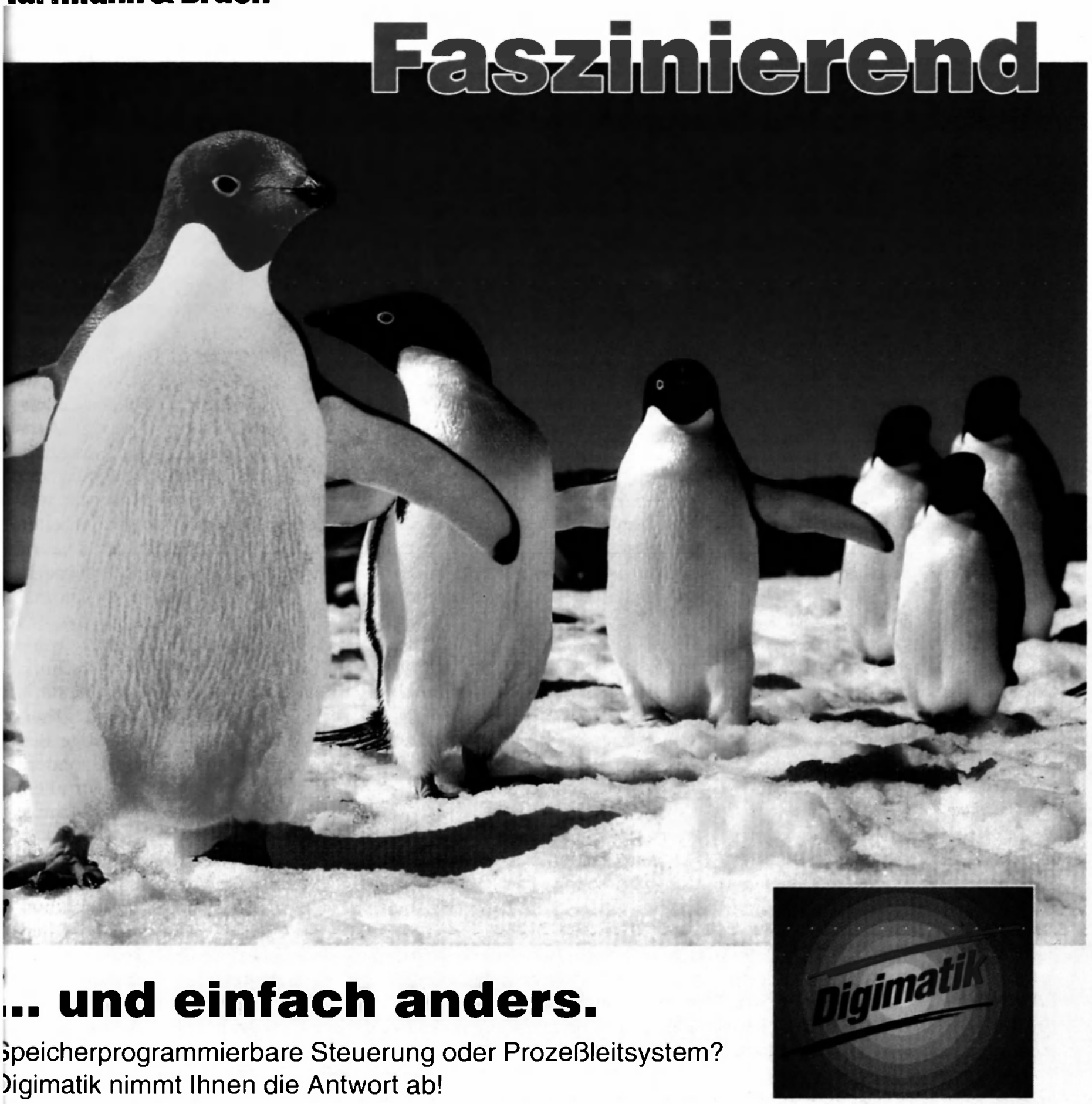

ie fordern eine komfortable Unterstützung bei Automatisierungsaufgaben mit Bedienung und leobachtung - wie es bei Prozeßleitsystemen üblich ist. Auch die gewohnten Vorzüge einer peicherprogrammierbaren Steuerung - wie normierte Programmiersprachen und kostenünstige Hardware - möchten Sie nicht missen.

ligimatik, das neue Kompakt-Leitsystem, bietet beides. Das durchgängige Systemkonzept arantiert eine hohe Effektivität bei Konfiguration, Inbetriebnahme und Wartung und verringert rastisch den Engineeringaufwand.

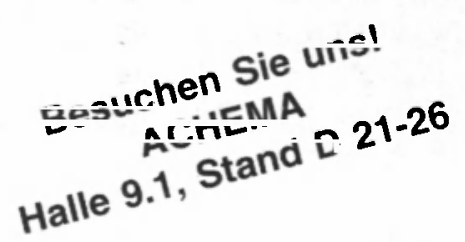

Venn Sie sich für das ,andere“ Leitsystem interessieren,

Jllten Sie weitere Informationen anfordern: 